\title{
Signal Formation in Irradiated Silicon Detectors
}

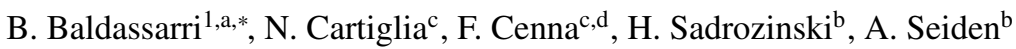 \\ ${ }^{a}$ University College London, Gower St, WC1E 6BT, London \\ ${ }^{b}$ University of California, Santa Cruz, Ca 95064, USA \\ ${ }^{c}$ INFN - Torino, Italy \\ ${ }^{d}$ Università di Torino, Italy
}

\begin{abstract}
In this paper we present an initial study on the effects induced by radiation on the signal generated by a minimum ionizing particle in silicon detector. The results are obtained by implementing in the simulation program Weightfield2 (WF2) charge carrier trapping and non linear distribution of the electric field. Results of sample simulations are presented, along with a discussion of the limitations of the current approach and ideas for future improvements.
\end{abstract}

Keywords: Double Junction, Silicon Sensors, Simulation, Trapping

\section{Introduction}

Radiation damage in silicon detectors results into three main changes of the detector performance: (i) a variation of the effective doping concentration and distribution, (ii) an increase 5 in the leakage current, and (iii) a decrease in the charge collec- ${ }^{35}$ tion efficiency. These effects are the measurable consequences of the creation of defects in the silicon lattice, which act as either sources or sinks of charge carriers. The defects created are assumed to scale linearly with the amount of energy deposited 10 into displacements, and the damage created by any kind of particle has been related to that of a $1 \mathrm{MeV}$ neutron trough the use of a hardness factor. Nevertheless, defect generation is still considerably particle dependent. While any impact particle having a sufficiently high energy to create a primary knock-on atom 15 leads to the creation of simple point defects, neutral hadron irradiation is also largely responsible for the production of cluster type defects. Such microscopic differences have a large impact on the macroscopic properties mentioned at the beginning of the section. [1] Many works have already proposed a parametrisa20 tion of the change in the detector's macroscopic properties in function of the fluence. The trapping induced decrease of signal has been modelled following an exponential fashion [2] [3] [4]:

$$
I=I_{0} e^{\frac{-t}{\tau_{e f f}}}
$$

where $\tau_{\text {eff }}$ refers to the effective trapping time, which is inversely proportional to the fluence $\phi$ :

$$
\frac{1}{\tau_{e f f}}=\beta \phi
$$

The leakage current, on the other hand, has been observed to have a linear dependance on the fluence [5]:

$$
I_{\text {leak }}=\alpha(t, T) V \phi
$$

\footnotetext{
${ }^{*}$ Corresponding author

Email address: bianca.baldassarri.12@ucl.ac.uk (B. Baldassarri)
}

with $\alpha$ being the leakage current damage constant, and $\mathrm{V}$ the volume of the detector. The evolution of the effective doping concentration has been found to be more complex. Not only donor and acceptor like defects are introduced changing the effective dopant concentration, but the dopant distribution itself evolves to be no longer uniform. The result is a change of the electric field from linear to quadratic, and the appearance of the so called double junction.

\section{Weightfiled2}

WF2 is a simulation program aimed at describing the performance of silicon and diamond detectors. [6]. A graphical user interface allows for the input of several parameters, like the configuration of the detector (number of strips, doping layers, thickness, width and pitch) and the working temperature and voltages (bias and depletion). These are then used as starting points to determine the detector's operational characteristics. After the electric field distribution is derived from the depletion voltage using Poisson's equation, the energy released by an incoming particle, whose type is selected by the user, is calculated with the aid of GEANT4 libraries. The induced signal current is then derived from Ramo's theorem. The drift of the electron-hole pairs generated by each incident particle (the choice of more than one MIP is possible, and the impact point and angle are customisable from the GUI) is followed, with a precision selected by the user (in terms both of percentage of electron-hole pairs simulated and of time unit) and a velocity calculated with respect to the drift field, mobility and saturation velocity. Amongst the doping configuration selection, the possibility of simulating a sensor with internal charge multiplication is present. The gain, chosen by the user from the GUI, has an exponential dependency on both a multiplication coefficient (determined by the local electric field giving rise to the multiplication) and the distance travelled along the electric field. The output of the simulation consists of several plots displaying the 
components of both current (electrons, holes, and gain carriers) and electric field (Ex and Ey). An optional feature allows to simulate the response of both a broadband and a charge sensitive amplifier, and to visualise the oscilloscope's signal.120 [6].

\section{Implementation of radiation damage effects}

To fully simulate the performance of an irradiated detector three factors should to be taken into account: (i) the change in the electric field from linear to quadratic, (ii) the generation of the additional charge carriers that constitute the leakage current, and (iii) the trapping of the charge carriers leading to the ${ }_{130}$ reduction in charge collection efficiency. As WF2's primary aim is to simulate the pure signal form, rather than including any kind of additional noise, it has been deemed appropriate to only include the changes concerning the electric field and the current. Such changes have been addressed by introducing four free parameters $\left(\beta_{e}, \beta_{h}, N_{e f f}\right.$ and $N_{A} / N_{D}$, whose significance will be explained in the following paragraph) in the graphical user interface, all of which can be adjusted to match experimental values and subsequently employed to make predictions. ${ }_{140}$ Seeing that WF2 individually follows the drift of each charge carrier, trapping has been implemented following a Montecarlolike treatment, extrapolating the probability for a single electron and hole to be trapped each nanosecond from the exponential current decay outlined in the introduction:

$$
P_{\text {trapping }}=1-e^{-\beta \phi}
$$

The value of the parameter $\beta$ can be experimentally determined, and is usually found to differ between electrons and holes. The basis of the charge correction method for the determination of the effective trapping time lies in the assumption of an exponential decrease of charge with time. The integral of the induced current doesn't exhibit any saturation in an irradiated detector. It is in fact characterised by a rise at voltages above the full depletion voltage, where the higher electric field reduces the drift time, and thus the amount of trapped charge. The determination of the effective trapping time is achieved by correcting the measured induced currents with an exponential such ${ }_{155}$ that the charge obtained by integration of such induced currents is constant for all voltages above $V_{d e p l}$. [2]. In the literature, be found [2] [3] [4] highlighting a dependance of such parameter both on the irradiation type (neutral or charged hadrons), 160 and on the detector type (doping type as well as manufacturing process). For this reason, the parameter $\beta$ is left for the user to input (in units of $10^{16} \mathrm{~cm}^{2} \mathrm{~ns}^{-1}$ ). The basic idea would be to extrapolate the specific value from charge collection efficiency studies on the desired detector type, and use it to make predic-165 tions for different geometries and thicknesses, and for higher fluences. These predictions are expected to hold as long as two the traps within the detector bulk (a condition which is expect to

hold, as it is a fundamental assumption behind the entire treatment of trapping in this circumstance), and an independence of the parameter $\beta$ on the fluence. As mentioned in the introduction, the evolution of the electric field due to radiation damage is complex as it involves both changes in dopant concentration and distribution, and does not follow any directly recognizable pattern. As a consequence, the present approach towards the matter consists in deriving the electric field from a dopant concentration and distribution entirely user selected. This is carried out by offering a choice between a linear and a step dopant distribution throughout the detector's bulk in the graphical user interface, with the number of additional dopant atoms $N_{\text {eff }}$ (in units of $10^{12}$ ) and the ratio between donors and acceptors $N_{A} / N_{D}$ specified by the user. In this way it becomes possible to simulate various conformations of the field, and compare the signal from an alpha particle or from edge TCT with experimental data and extract the value of $N_{\text {eff }}$ and $N_{A} / N_{D}$ for a fluence and given detector type. While it is evident how the adopted strategy hardly provides any predictive power in terms of varying fluence or detector type, it nevertheless remains a valid tool for investigating the effects of a non linear electric field on a MIP signal. Once a higher amount of measurements has been performed, it could ideally be possible to determine $N_{e f f}$ and $N_{A} / N_{D} \beta_{e}$ and $\beta_{h}$ from comparison with experimental data for an array of fluences and detector types. A database could then be integrated in WF2, such that an accurate performance prediction would be obtained simply by specifying the type of the detector of interest and the amount of charged or neutral hadronic irradiation it is subject to. WF2 also offers the possibility of including acceptor creation by deep traps and initial acceptor removal [7]:

$$
N_{A}(\Phi)=g_{e f f} \Phi_{e q}+N_{A}(0) e^{-c_{2} \Phi_{e q}}
$$

where $N_{A}(0)$ is the initial acceptor number, $g_{\text {eff }}=0.02 \mathrm{~cm}^{-1}$ and $c_{2}=4 * 10^{-9} N_{A}(0)^{-0.4}$ is a factor that depends on the initial acceptor concentration (assuming complete initial acceptor removal).

\section{Simulation Results and Comparison to experimental data}

On the basis of the description above, various simulations have been conducted to examine the changes in the performance of irradiated detectors with different thicknesses and gain values, and some of the results are hereby presented. Using the values $\beta_{e}$ and $\beta_{h}$ derived in [2] for n-in-p neutron irradiated FZ detectors, charge collection efficiency curves have been produced which highlight the advantages of the choice of thinner detectors and of the presence of an extra gain layer.

Although when scaled to the full multiplied charge collected prior to irradiation the degradation of the signal due to trapping appears visibly more pronounced in Low Gain Avalanche Detectors (LGAD) [8] (Figure 1 left side), the signal yielded thanks to the presence of the extra gain layer still exceeds that of a conventional detector (Figure 1 right side) for all investigated fluences. Such result is considerably more pronounced in thin 
the general reduction in signal is more than halved for a $50 \mu \mathrm{m}$ sensor compared to the common $300 \mu \mathrm{m}$. Furthermore, the performance of thin LGADs suffers from a much less drastic reduction than thicker ones, which, for fluences of about $5 \cdot 10^{15} n_{e q} \mathrm{~cm}^{-2}$ seem to approach the efficiency of traditional devices.
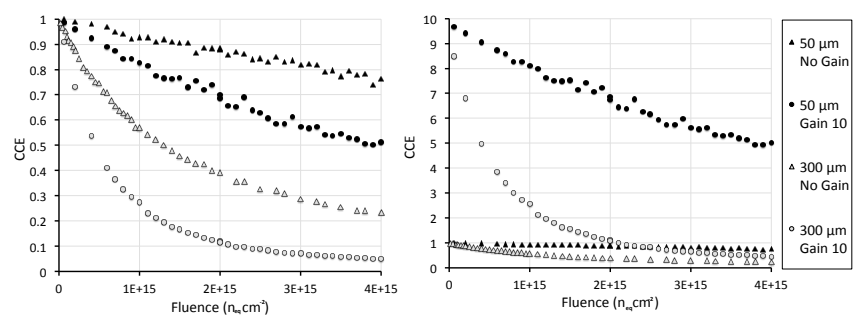

Figure 1: Comparison of the evolution of the charge collection efficiency as a function of fluence for detectors of different thicknesses and gain values. On the left side, the charge is normalised to unity for ease of comparison with traditional detectors, while on the right it is normalized to the charge collected by a sensor without gain.

Figure 2 and 3 display the evolution of the signal typically obtained from a traditional silicon detector and an LGAD. While the overall drift time remains unchanged, as predictable by considering that the only effect of trapping in the present simulation is a permanent elimination of charge carriers from the total current, a visible transformation in the signal shape is noticeable.

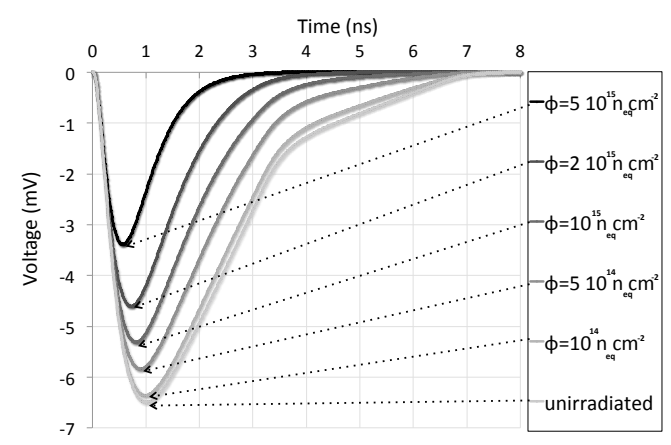

Figure 2: Evolution of the signal output of a traditional n-in-p silicon pad detector $\left(300 \mu \mathrm{m}\right.$, operated at $500 \mathrm{~V}$ and $300 \mathrm{~K}$, with $\left.V_{\text {depl }}=50 \mathrm{~V}\right)$ with irradiation. ${ }^{200}$ Only effects related to charge trapping are included

To understand the implications of such change in shape on the timing performance of LGAD sensors, it's essential to first ${ }_{205}$ examine the factors determining the time resolution $\sigma_{t}[9]$ : time walk, jitter, and TDC binning:

$$
\sigma_{t}^{2}=\left(\left(\frac{V_{t h}}{S / t_{r}}\right)_{R M S}\right)^{2}+\left(\frac{N}{S / t_{r}}\right)^{2}+\left(\frac{T D C_{\text {bin }}}{\sqrt{12}}\right)^{2}
$$

where $V_{t h}$ indicates the comparator threshold $\mathrm{S}$ the signal amplitude, $t_{r}$ the rise time, and $\mathrm{N}$ the noise. The first two terms in above equation carry a clear dependence on the signal to rise time ratio, and indicate the necessity for a fast rise time (as well

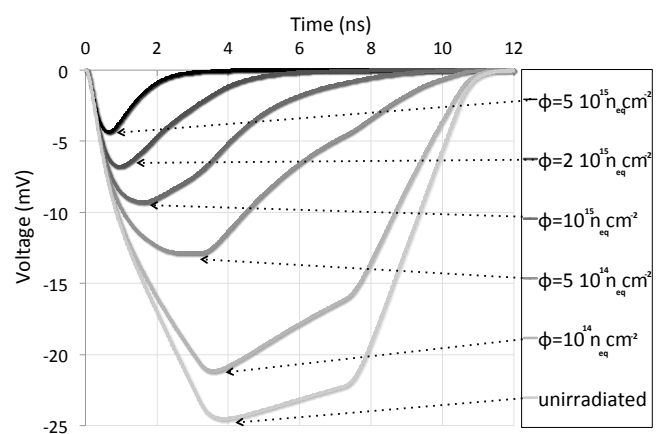

Figure 3: Evolution of the signal of an p-bulk LGAD silicon pad detector $\left(300 \mu \mathrm{m}\right.$, operated at $500 \mathrm{~V}$ and $300 \mathrm{~K}$, with $\left.V_{d e p l}=50 \mathrm{~V}\right)$ with irradiation. Only effects related to charge trapping are included

as a low electronic noise), which is one of the main features that appears to have a significant worsening in irradiated detectors. This is nevertheless also a feature showing a dramatic improvement in thin detectors, as clearly visible by comparing Figure 3 and 4

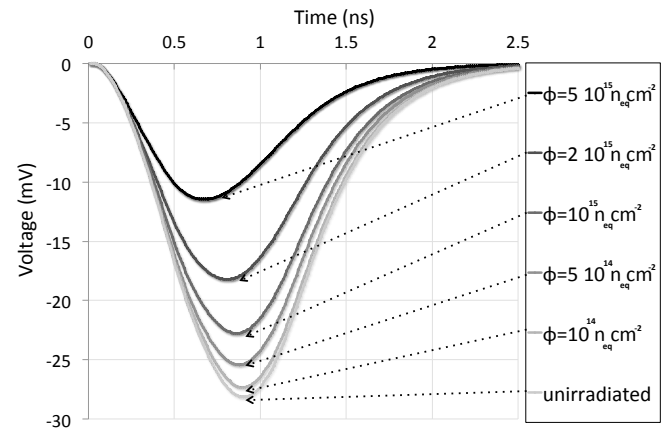

Figure 4: Evolution of the signal from a broadband amplifier of a p-bulk ultrafast silicon pad detector $\left(50 \mu \mathrm{m}\right.$, operated at $250 \mathrm{~V}$ and $300 \mathrm{~K}$, with $\left.V_{d e p l}=10 \mathrm{~V}\right)$ with irradiation. Only effects related to charge trapping are included

WF2 can also be used to investigate the effects of non linear electric fields such are those generated by the so called double-junction mechanism. A comparison of the MIP signal between an unirradiated detector and one subject to flunce $\Phi=5 \cdot 10^{15} n_{e q} \mathrm{~cm}^{-2}$ is shown in Figure 5, where the corresponding electric field results from a choice of a linear distribution of $3 \cdot 10^{13}$ additional dopant atoms, with a ratio between donors and acceptors of 0.5 , and, once again, the $\beta$ parameters derived in [2]. Figure 5 shows a few interesting characteristics of the signal: a fast signal rise time, and the fact that in the presence of a double-junction the height of the signal is nearly unaffected, although a reduction in the total collected charge is evident. Both features seem to indicate little disruptive consequences in the introduction of a double junction, but this is far from being conclusive evidence: the effect on the noise has not been addressed, and so hasn't the presence of a field free region between the two "junctions" (a region where donors and acceptors balance out so that there is no net charge), which would 
result in a much more significant loss of charge.

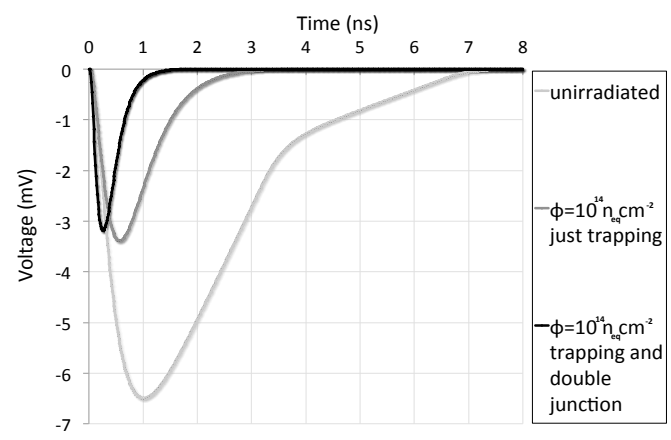

Figure 5: Comparison of the signal from a n-in-p silicon pad detector $(300 \mu \mathrm{m}$, operated at $500 \mathrm{~V}$ and $300 \mathrm{~K}$, with $V_{\text {dell }}=50 \mathrm{~V}$ ) between unirradiated and irradiated with $\Phi=5 \cdot 10^{15} n_{e q} \mathrm{~cm}^{-2}$, where the latter case has been simulated adding only trapping effects, or trapping and the effect of a quadratic electric field

\section{Future Developments}

It's important to notice that the simulations presented so far don't provide a complete description of the behaviour of an irradiated detector. A change that hasn't been addressed is that 270 of the carrier mobility. Trapping modifies the signal in that it reduces the number of charges reaching the electrodes, which is the main effect for what concerns the charge collection efficiency. The carrier mobility, on the other hand, impacts, along-275 side the electric field distribution, the shape of the signal itself, which is of major importance when dealing with timing applications. The main reason for the absence of this influent modification is the absence, at the present moment, of a 280 widely accepted parametrisation of the dependance of mobility on fluence in the literature (past proposals are in contrast with each other [10] [11]). A second pressing issue is that concerning the influence of the applied voltage on the current degra-285 dation: for high voltages and fluences a parametrisation of the trapping time purely as a function of fluence no longer provides a good description without a second a voltage dependent (or rather, electric field dependent) term. [12]. Charge multiplica-290 tion effects have also been observed for high fluences and voltage [13], however it's difficult to find a general parametrization of the effect with the available data.

\section{Conclusion}

The article presents an update to the simulation program Weightfield2, adressing the effects that charge trapping and the presence of a quadratic electric field have on irradiated silicon detectors. A trapping model based on an exponential decay of the current output has been included, along with a choice between constant, linear and step dopant distribution in the detector bulk. The analysis of the simulations produced up to the present highlights the convenience in the choice of thin detectors, and in the presence of an internal gain mechanism. The superior timing efficiency of LGADs seems not to be drastically compromised as the the signal rise time is not particularly impacted by the presence of a non linear electric field. The significant decrease in charge collection efficiency shown by both traditional sensors and LGADs can be largely contained by the employment of thin detectors. Charge trapping, in fact, appears to have up to $50 \%$ less impact on $50 \mu \mathrm{m}$ sensors than it has on the standard $300 \mu \mathrm{m}$ ones. The simulations presented are not entirely conclusive, as factors like the change in the carrier mobility and the presence of radiation-induced charge multiplication have yet to be implemented, as well as the effect of the electric field strength on charge trap distribution.

\section{Acknowledgements}

We Acknowledge the following funding agencies: Horizon 2020 under the program ERC Adv Grants 669529; Ministero degli Affari Esteri, Italy, MAE, U.S. Department of Energy grant number DE-SC0010107.

\section{References}

[1] J. Lange, Radiation damage in proton irradiated epitaxial silicon detectors, Physikalische Diplomarbeit, Universitat Hamburg.

[2] G. Kramberger, et al., Effective trapping time of electrons and holes in different silicon materials irradiated with neutrons, protons and pions, $\mathrm{Nu}-$ clear Instruments and Methods in Physics Research A 481 (2002) 297305.

[3] J. Lange, et al., Charge collection studies of proton-irradiated n- and p-type epitaxial silicon detectors, Nuclear Instruments and Methods in Physics Research A 624 (2010) 405-409.

[4] J. Weber, et al., Free charge carriers trapping properties in neutronirradiated dofz silicon pad detectors, IEEE Transactions On Nuclear Science 54-6 (2007) 2071-2075.

[5] G. Kramberger, et al., Comparison of pad detectors produced on different silicon materials after irradiation with neutrons, protons and pions, $\mathrm{Nu}-$ clear Instruments and Methods in Physics Research A 612 (2010) 288295.

[6] F. Cenna, et al., Weightfield2: a fast simulator for silicon and diamond solid state detector, Nuclear Instruments and Methods in Physics Research A, In Press.

[7] G. Kramberger, et al., Radiation effects in Low Gain Avalanche Detectors after hadron irradiations, JINST 10 (07) (2015) P07006. doi:10.1088/ 1748-0221/10/07/P07006

[8] G. Pellegrini, et al., Technology developments and first measurements of low gain avalanche detectors (lgad) for high energy physics applications, Nucl. Instrum. Meth. 765A (2014) 12-16.

[9] N. Cartiglia, et al., Design optimization of ultra-fast silicon detectors, Nucl. Instrum. Meth. A796 (2015) 141-148. doi:10.1016/j.nima. 2015.04 .025

[10] J.V.Vaitkus, et al., Analysis of electron mobility dependence on electron and neutron irradiation in silicon, Presented at the 26th RD50.

[11] Drift mobility and electric field in silicon detectors irradiated with neutrons and protons up to $1017 \mathrm{neq} / \mathrm{cm} 2$, Presented at the 2015 IEEE Nuclear Science Symposium San Diego, CA.

[12] J. Lange, et al., Charge collection studies of proton irradiated n- and p-type epitaxial silicon detectors, Nuclear Instruments and Methods in Physics Research A 624 (2010) 405?409.

[13] Charge multiplication in highly irradiated planar silicon sensors, Procedings of Science. 